\title{
The Weighted GMD Model for multiplicity distributions
}

\section{Probing gluon production at LHC energies}

\author{
W.Y.Wang ${ }^{1, a}$, S.Seah ${ }^{1}$, J.Setianegara ${ }^{1}$, A.H.Chan ${ }^{1}$, and C.H.Oh ${ }^{1}$ \\ ${ }^{1}$ Physics Department, National University of Singapore, Singapore
}

\begin{abstract}
A new distribution, the Weighted GMD (WGMD) is obtained from the Generalised Multiplicity Distribution (GMD), describing charged-particle multiplicity distributions as the hadronisation products of quark and gluon branching with fluctuations in the initial gluon numbers produced from the collision. The WGMD is shown to describe charged-particle multiplicity distributions in $p p$ collisions at the Large Hadron Collider (LHC), and the average initial gluon number is obtained for Poisson distributed gluon multiplicities.
\end{abstract}

\section{Introduction}

Multiplicity distributions reveal useful information on the characteristics of particle production processes in high energy collisions. The shape of multiplicity distributions reveals whether the production of the particles within the emission region are correlated. A Poisson distributed (PD) multiplicity for example, indicates the independent emission of single particles. Cascades of particles originating from an initial number of ancestor particles produced after the collision, lead to a broader distribution like the Negative Binomial Distribution (NBD) [1].

Like the NBD, the Generalised Multiplicity Distribution (GMD) [2-4] can be derived from a very similar cascade process. The GMD is a solution of a stochastic branching equation [5] describing the evolution of quarks and gluons as Markov branching processes. This intuitive picture has been shown to be equivalent to an algorithm calculating the multi-parton distributions within a QCD jet in a leading logarithmic approximation [6], and yields the GMD and NBD as solutions.

Given $m$ and $n$ number of quarks $(q)$ and gluons $(g)$ respectively, the dominant processes of quark bremsstrahlung $(q \rightarrow q+g)$ and gluon fission $(g \rightarrow g+g)$ result in a shower of quarks and gluons which eventually hadronise into the observed particles. In this picture, the stochastic branching equation [5]

$$
\frac{\partial P_{m, n}(t)}{\partial t}=-\tilde{A} m P_{m, n}(t)+\tilde{A} m P_{m, n-1}(t)-A n P_{m, n}(t)+A(n-1) P_{m, n-1}(t),
$$

relates the probability of obtaining $m$ quarks and $n$ gluons $\left(P_{m, n}\right)$ from $m$ quarks and $n$ or $n-1$ gluons at each branching step. $A \Delta t$, and $\tilde{A} \Delta t$ are the probabilities of quark bremsstrahlung and gluon fission

ae-mail: w.y.wang@u.nus.edu 
within the infinitesimal interval $\Delta t$. The QCD evolution parameter $t$ is given by

$$
t=\frac{6}{11 N_{c}-2 N_{f}} \ln \left[\frac{\ln \left(Q^{2} / \mu^{2}\right)}{\ln \left(Q_{0}^{2} / \mu^{2}\right)}\right]
$$

where $Q$ is the initial parton $(q / g)$ invariant mass, $Q_{0}$ is the hadronisation mass, $\mu$ is a QCD mass scale of a few GeV, $N_{c}$ is the number of colours, and $N_{f}$ is the number of flavours.

For an initial $m$ and $k^{\prime}$ number of quarks and gluons, the solution of equation 1 yields the final multiplicity of $n$ partons which hadronise to form $n$ particles distributed according to the GMD:

$$
P_{G M D}\left(n ; p, k, k^{\prime}\right)=\frac{\Gamma(n+k)}{\Gamma\left(n-k^{\prime}+1\right) \Gamma\left(k^{\prime}+k\right)}(1-p)^{n-k^{\prime}}(p)^{k^{\prime}+k},
$$

where $k=m \tilde{A} / A$ and $p=\exp (-A t)=\left(k^{\prime}+k\right) /(\bar{n}+k)$. The average multiplicity of final state hadrons $\bar{n}$ is controlled by the initial quark and gluon numbers as well as the branching probability through

$$
\bar{n}=\frac{k^{\prime}+k}{p}-k
$$

The GMD is then a more general solution as the name suggests, in the sense that it encompasses to the Fury-Yule Distribution [7] for which $k=0$, and the NBD where $k^{\prime}=0$. Furthermore, the NBD converges to the PD for $k \rightarrow \infty$.

The GMD has been successfully applied to describe charged-particle multiplicity distributions from $e^{+} e^{-}, p p$, and $p \bar{p}$ collisions [2-4]. However it increasingly experiences a difficulty due to the emergence of a kink or "shoulder" like structure in charged-particle multiplicity data above $\sqrt{s}=900$ $\mathrm{GeV}[8,9]$ in both $p p$ and $p \bar{p}$ collisions, as well as a spike in the $n=0$ probability. Theoretically, the hadron production mechanism and hadronisation in the GMD only allows for $n \geq k^{\prime}$, and poses a difficulty in the low multiplicity and high energy regime.

In brief, this paper proposes a modified GMD with a more realistic description of the initial state of the particle emission source. In addition, this proposed modified GMD achieves a better fit of data from the CMS collaboration at 0.9, 2.36 and $7 \mathrm{TeV}$ [9] with the same number of parameters, and provides a physical interpretation of the extracted model parameters.

\section{The weighted GMD model}

The weighted GMD (WGMD) model is obtained from the GMD by introducing event-by-event fluctuations in the production of initial gluons. If each independent collision event emits $k^{\prime}$ number of gluons distributed according to a distribution $P\left(k^{\prime} ; x_{1}, \ldots, x_{r}\right)$ with $r$ parameters, then the WGMD is given by

$$
P_{W G M D}\left(n ; x_{1}, \ldots, x_{r}, p, k\right)=\sum_{k^{\prime}=0}^{n} P\left(k^{\prime} ; x_{1}, \ldots, x_{r}\right) \times P_{G M D}\left(n ; p, k, k^{\prime}\right) .
$$

The observed final state multiplicity distribution is thus a weighted sum of the GMD over the probabilities of the initial states. The summation is done for $k^{\prime}=0$ to $n$ as those are the only physical possibilities since the number of gluons cannot exceed the number of final state hadrons. The gluons are assumed to go through a cascade process and then eventually split into quarks and gluons that hadronise (i.e. a 1-1 correspondence between the gluon number right before hadronisation, and the hadron number right after). 
Without specifying the form of the weight $P\left(k^{\prime} ; x_{1}, \ldots, x_{r}\right)$, the WGMD can be shown to be normalised and the mean, $\bar{n}=\sum_{n=0}^{\infty} n P_{W G M D}\left(n ; x_{1}, \ldots, x_{r}, p, k\right)$, given by

$$
\bar{n}=\frac{\left\langle k^{\prime}\right\rangle+k}{p}-k
$$

where $\left\langle k^{\prime}\right\rangle=\sum_{k^{\prime}=0}^{\infty} k^{\prime} P\left(k^{\prime} ; x_{1}, \ldots, x_{r}\right)$. Equation 6 holds the same form as the mean of GMD expressed by equation 4 , except with $k^{\prime}$ replaced by $\left\langle k^{\prime}\right\rangle$. Detailed calculations are provided in the appendix.

Perhaps the simplest manifestation of the WGMD is where the weight is Poisson distributed, i.e. $P\left(k^{\prime} ; \overline{k^{\prime}}\right)=\overline{k^{\prime}} k^{\prime} \exp \left(-\overline{k^{\prime}}\right) / k^{\prime}$ !, where $\overline{k^{\prime}}$ is the average number of gluons. From equation 6 , the mean of $n$ with the Poisson weighted GMD (PGMD) is simply given by $\bar{n}=\frac{\overline{k^{\prime}}+k}{p}-k$, where $\left\langle k^{\prime}\right\rangle$ is replaced by the Poisson average $\overline{k^{\prime}}$. The Poisson weight in the WGMD represents the independent production of single gluons in the initial state of QCD evolution after collision and the parameter $\overline{k^{\prime}}$ represents the average gluon number over all collision events.

The number of initial gluons $k^{\prime}$ is often said to be able to take non-integral values if interpreted in an average sense [2-4]. The WGMD describes an ensemble of events with varying gluon numbers described by the weight factor. This model opens up possibilities to probe the average gluon number as a function of the collision centre-of-mass energy and pseudorapidity acceptance. Given a different weight distribution (e.g. NBD), correlations in gluon production can also be studied and compared to the Poisson case.

\section{Results}

Figure 1 exhibits a comparison of 3 different PGMD with parameters $k=0.1,1$, and 5. The other parameters are fixed at $p=0.1$ and $\overline{k^{\prime}}=2$. For a smaller $k$, the distribution also peaks at smaller $n$. At $k=0.01$, the distribution has a spike at $n=0$. For a relatively larger $k$, varying the parameters $p$ and $\overline{k^{\prime}}$ does not reproduce the spike while for a small $k$, the variation of $p$ and $\overline{k^{\prime}}$ only modulates the height of the spike.

Figures 2-4 show the multiplicity distributions measured by the CMS collaboration [9] at centreof-mass energies of $0.9,2.36$, and $7 \mathrm{TeV}$. Comparison is made with the best fit PGMD and GMD. The interior point algorithm $[10,11]$ is used to find the parameters that give the minimum $\chi^{2}$ fit to the data, excluding point $n=0$. The parameters that give the best fit distributions are shown in table 1 .

The $\chi^{2} / d o f$ show that the PGMD describes the multiplicity distributions better than the GMD. The PGMD describes the tail ends of the distribution better than the GMD, but not as well at low multiplicities.

From table 1, it is evident that the GMD that best describes the data reduces to the NBD $\left(k^{\prime}=0\right)$. Given the computation of $\chi^{2}$ which considers the points $n \geq 1$, we have the constrain of $k^{\prime} \leq 1$. The characteristic feature of the GMD having an initial condition of $k^{\prime}$ number of gluons that branch and eventually hadronise, ensure that there are at least the same number of $n$ hadrons as the initial number of gluons. This constraint limits the applicability of the GMD in finding an optimised solution.

The PGMD does not have this constraint since it considers a spread of initial conditions that describe Poisson processes which produce the gluons. At $0.9,2.36$, and $7 \mathrm{TeV}$, the model gives $1.07,0.741$, and 0.865 gluons respectively, on average. For increasing centre-of-mass energies, the parameter $p$ decreases monotonically from 0.135 to 0.0614 . From equation 2 and the definition of $p$, the model describes an increasing initial parton invariant mass $Q$ as a function of the centre-of-mass energy, given a fixed branching probability $A$. The corresponding mean hadron numbers 18.1, 23.3, and 30.4 calculated using equation 6 is compatible with the experimental values measured by the CMS collaboration [9]. 


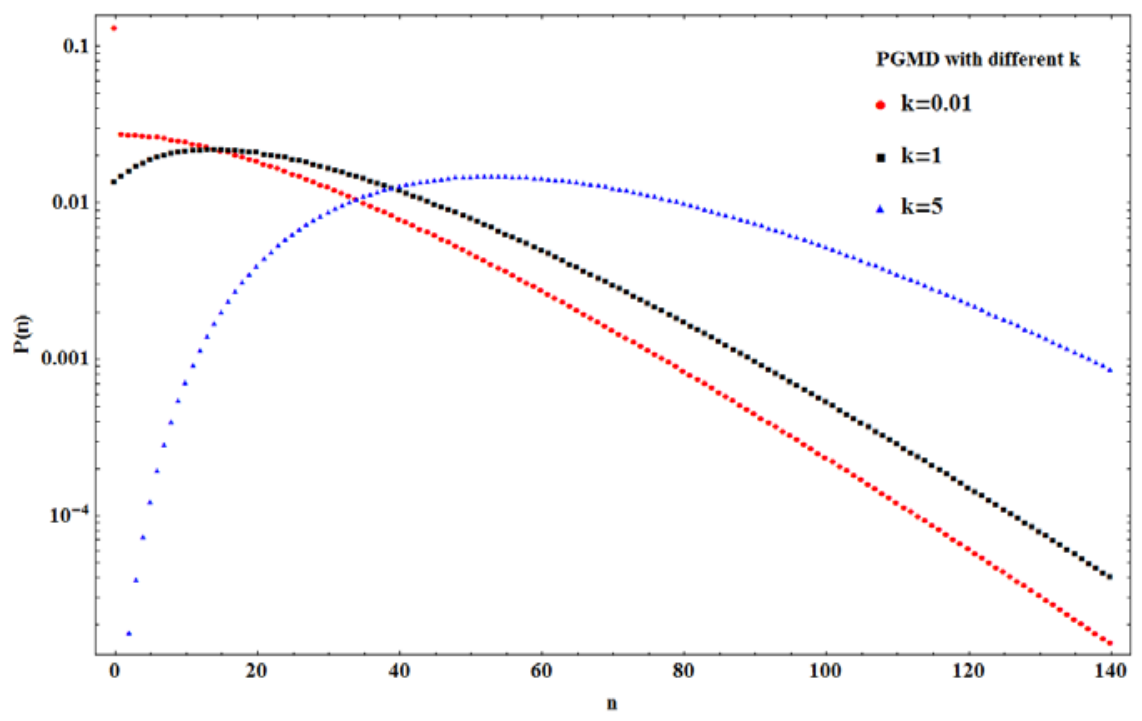

Figure 1. Comparison of PGMD with different $k$ values.

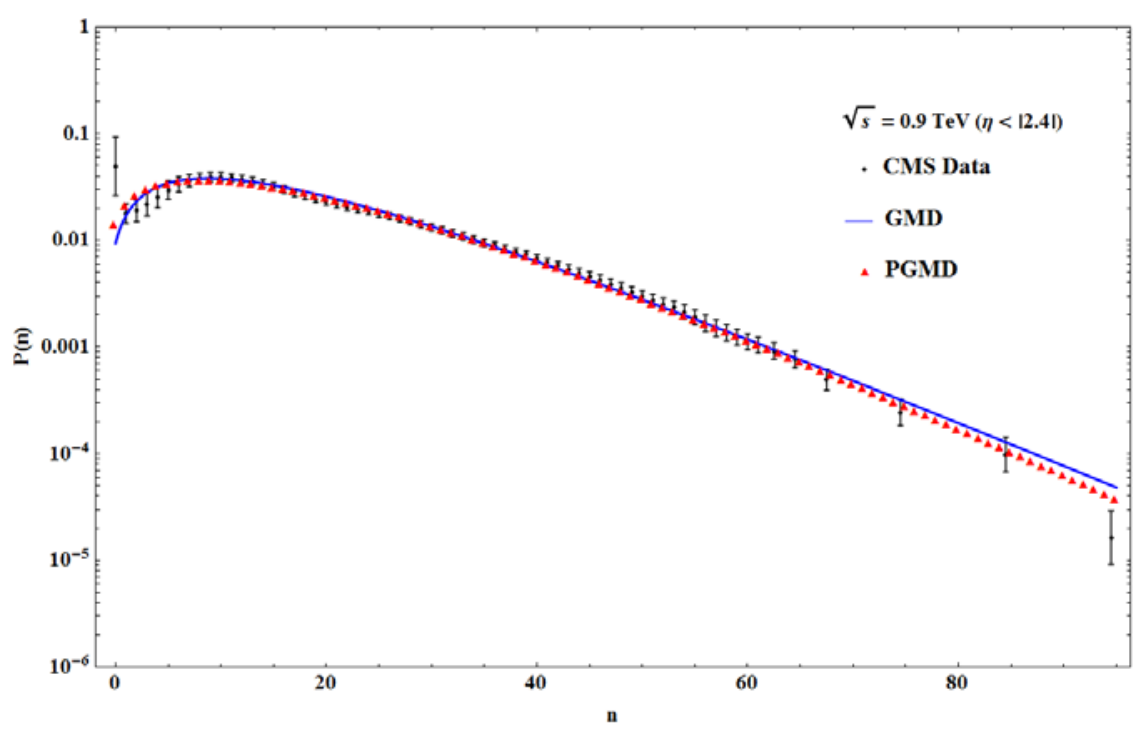

Figure 2. Charged-particle multiplicity distribution at $\sqrt{s}=0.9 \mathrm{TeV}$ and $|\eta|<2.4$ compared to the best fit PGMD (triangle) and GMD (line). The vertical lines in the data points represent the statistical errors and systematic uncertainties added in quadrature. 


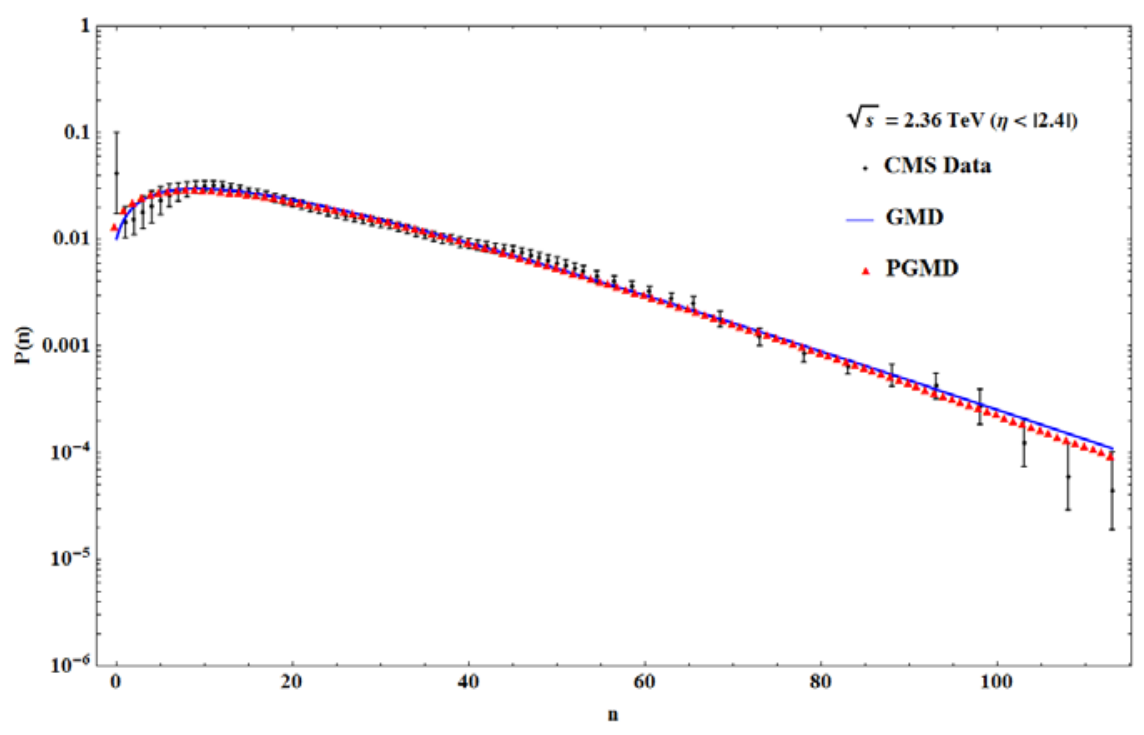

Figure 3. Charged-particle multiplicity distribution at $\sqrt{s}=2.36 \mathrm{TeV}$ and $|\eta|<2.4$ compared to the best fit PGMD and GMD. The markers used and description of error bars are the same as in Fig. 2.

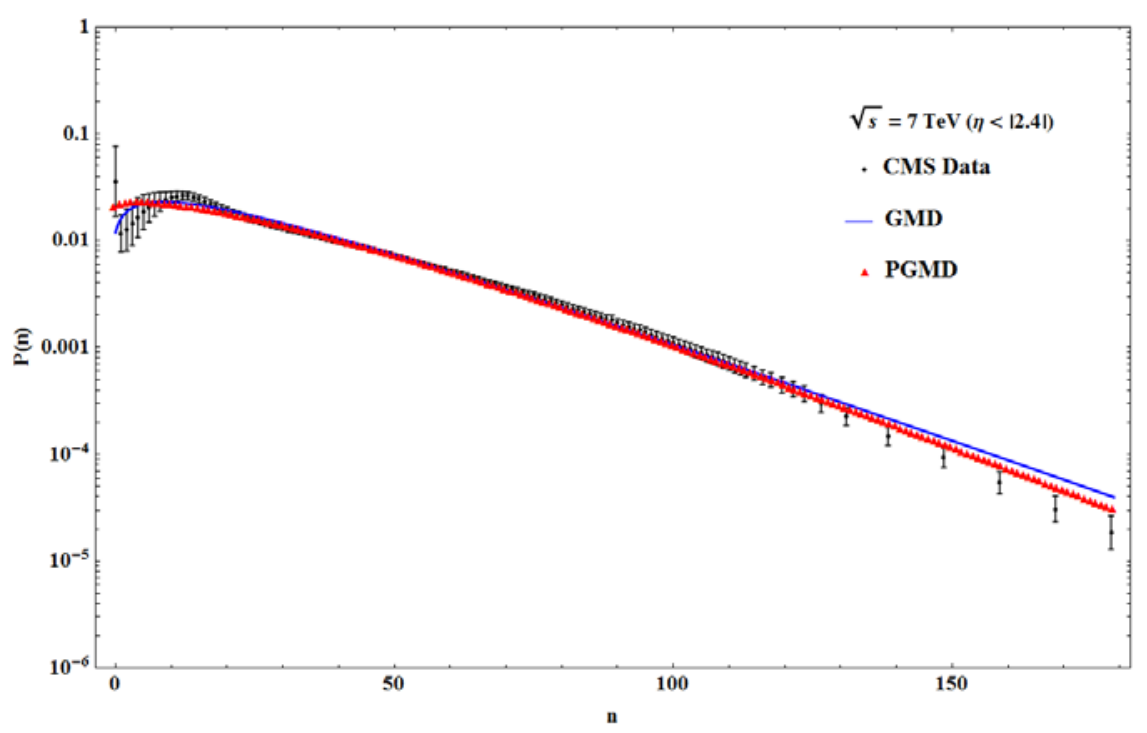

Figure 4. Charged-particle multiplicity distribution at $\sqrt{s}=7 \mathrm{TeV}$ and $|\eta|<2.4$ compared to the best fit PGMD and GMD. The markers used and description of error bars are the same as in Fig. 2. 
Table 1. Summary of the best fit parameter values.

\begin{tabular}{|c|c|c|c|c|}
\hline & $\sqrt{s}(\mathrm{TeV})$ & 0.9 & 2.36 & 7 \\
\hline \hline \multirow{4}{*}{ PGMD } & $p$ & 0.135 & 0.0904 & 0.0614 \\
\cline { 2 - 5 } & $k$ & 1.59 & 1.50 & 1.07 \\
\cline { 2 - 5 } & $\overline{k^{\prime}}$ & 1.07 & 0.741 & 0.865 \\
\cline { 2 - 5 } & $\chi^{2} /$ dof & $36.7 / 65$ & $36.9 / 67$ & $93.4 / 124$ \\
\hline \multirow{4}{*}{ GMD } & $\bar{n}$ & 18.4 & 23.4 & 31.0 \\
\cline { 2 - 5 } & $k$ & 2.02 & 1.71 & 1.41 \\
\cline { 2 - 5 } & $k^{\prime}$ & 0.00 & 0.00 & 0.00 \\
\cline { 2 - 5 } & $\chi^{2} /$ dof & $47.1 / 65$ & $41.3 / 67$ & $136 / 124$ \\
\hline
\end{tabular}

\section{Conclusion}

The weighted Generalised Multiplicities Distribution model is obtained from a weighted sum of the Generalised Multiplicities Distribution. The model describes a mixed distribution with weights corresponding to the likelihood of the initial production of a certain gluon multiplicity. This model provides a more realistic description of collision and production conditions where the initial gluon number fluctuates on an event by event basis. The normalisation of the distribution is shown and the mean is derived.

A specific realisation of the model has weights described by a Poisson distribution of the gluon multiplicity. This Poisson weighted Generalised Multiplicities Distribution is applied to chargedparticle multiplicity distributions measured by the CMS collaboration. Although the "shoulder" in the observed multiplicity distribution is still not reflected by the model, it describes the data better than the pure Generalised Multiplicities Distribution. The parameters that give the best fit are obtained, and the mean multiplicity given by the model is compatible with the experimentally observed values.

The application of Poisson weights to describe the gluon number production implies that the sources produce independent and uncorrelated single gluons. The use of other distributions as weights, for example the Negative Binomial Distribution, may be feasible and would suggest a different picture where the gluons are produced in a correlated manner. Insight on gluon production may be obtained by the comparison of such distributions.

\section{References}

[1] A. Giovannini, L. Van Hove, A. Phys. Pol. B 19, 495 (1988)

[2] C.K. Chew, D. Kiang, H. Zhou, Phys. Lett. B 186, 411 (1987)

[3] A.H. Chan, C.K. Chew, Phys. Rev. D 41, 851 (1990)

[4] A.H. Chan, C.K. Chew, Z. Phys. C 55, 503 (1992)

[5] A. Giovannini, Nucl. Phys. B 161, 429 (1979)

[6] K. Konishi, A. Ukawa, G. Veneziano, Nucl. Phys. B 157, 45 (1979)

[7] R.C. Hwa, C.S. Lam, Phys. Lett. B 173, 346 (1986)

[8] UA5 Collaboration, Z. Phys. C 43, 357 (1989)

[9] CMS Collaboration, JHEP 01, 79 (2011)

[10] Wolfram Research, Inc., Mathematica, version 9.0 edn. (Wolfram Research, Inc., Champaign, Illinois, 2012)

[11] S. Mehrotra, SIAM J. Optim. 2, 575 (1992) 